\title{
Quantum Phases of the Shastry-Sutherland Kondo Lattice: Implications for the Global Phase Diagram of Heavy-Fermion Metals
}

\author{
J. H. Pixley, ${ }^{1, *}$ Rong Yu, ${ }^{2,1}$ and Qimiao $\mathrm{Si}^{1}$ \\ ${ }^{1}$ Department of Physics and Astronomy, Rice University, Houston, Texas 77005, USA \\ ${ }^{2}$ Department of Physics and Beijing Key Laboratory of Opto-Electronic Functional Materials \\ and Micro-Nano Devices, Renmin University of China, Beijing 100872, China
}

(Received 27 December 2013; published 24 October 2014)

\begin{abstract}
Considerable recent theoretical and experimental effort has been devoted to the study of quantum criticality and novel phases of antiferromagnetic heavy-fermion metals. In particular, quantum phase transitions have been discovered in heavy-fermion compounds with geometrical frustration. These developments have motivated us to study the competition between the Ruderman-Kittel-Kasuya-Yosida and Kondo interactions on the Shastry-Sutherland lattice. We determine the zero-temperature phase diagram as a function of magnetic frustration and Kondo coupling within a slave-fermion approach. Pertinent phases include the valence bond solid and heavy Fermi liquid. In the presence of antiferromagnetic order, our zero-temperature phase diagram is remarkably similar to the global phase diagram proposed earlier based on general grounds. We discuss the implications of our results for the experiments on $\mathrm{Yb}_{2} \mathrm{Pt}_{2} \mathrm{~Pb}$ and related compounds.
\end{abstract}

DOI: 10.1103/PhysRevLett.113.176402

PACS numbers: 71.10.Hf, 71.27.+a, 75.20.Hr

Geometrical frustration in insulating quantum antiferromagnets can lead to a variety of quantum phases, such as valence bond solids (VBSs) and quantum spin liquids [1]. Recent studies have discovered intriguing properties in a growing list of metallic systems with local magnetic moments residing on frustrated lattices. In these heavyfermion compounds, the interplay of Kondo screening and magnetic frustration may give rise to entirely new ground states and quantum phase transitions [2]. For example, the compounds $\mathrm{Yb}_{2} \mathrm{Pt}_{2} \mathrm{~Pb}[3]$ and $\mathrm{CePd}_{1-x} \mathrm{Ni}_{x} \mathrm{Al}$ [4] have spin$1 / 2$ local moments located on the Shastry-Sutherland and kagome lattices, respectively. Likewise, both $\mathrm{YbAgGe}$ [5] and $\mathrm{YbAl}_{3} \mathrm{C}_{3}$ [6] feature triangular lattices. All these compounds show an enhanced specific heat coefficient, implying a large effective mass and the presence of the Kondo effect.

General theoretical considerations of the competition between Kondo and Ruderman-Kittel-Kasuya-Yosida (RKKY) interactions have led to a proposal for the global phase diagram of heavy-fermion metals as a function of frustration or quantum fluctuations $(G)$, and the Kondo coupling $\left(J_{K}\right)[7,8]$; see Fig. 1(a). This phase diagram incorporates not only antiferromagnetic (AF) order, but also the physics of Kondo destruction [9-11]. From the Kondo-destroyed antiferromagnetic phase $\left(\mathrm{AF}_{S}\right)$, the transition to the heavy Fermi liquid phase $\left(P_{L}\right)$ could take place directly (type I), via the spin-density-wave phase $\left(\mathrm{AF}_{L}\right)$ (type II), or through the Kondo-destroyed paramagnetic phase $\left(P_{S}\right)$ (type III). The heavy-fermion compounds $\mathrm{CeCu}_{6-x} \mathrm{Cu}_{x}, \mathrm{YbRh}_{2} \mathrm{Si}_{2}$, and $\mathrm{CeRhIn}$ h have shown strong evidence for realizing the type I transition [12-15]. $\mathrm{CePd}_{3} \mathrm{Si}_{20}$, which is cubic and therefore would have a smaller $G$, has properties consistent with a type II transition [16]. Geometrical frustration is expected to enhance the quantum fluctuation parameter $G$, raising the prospect of realizing a type III transition. There is a recent surge of heavy-fermion materials that appear to be suitable for exploring this large- $G$ portion of the global phase diagram. In particular, $\mathrm{Yb}_{2} \mathrm{Pt}_{2} \mathrm{~Pb}$ and its homologues such as $\mathrm{Ce}_{2} \mathrm{Pt}_{2} \mathrm{~Pb}$ [3], featuring the geometrically frustrated Shastry-Sutherland lattice, may involve an intermediate VBS $P_{S}$ phase.

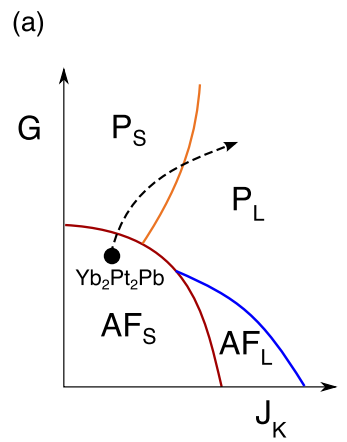

(b)

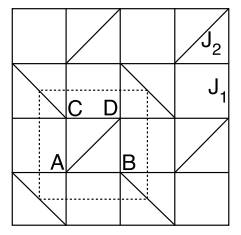

(c)

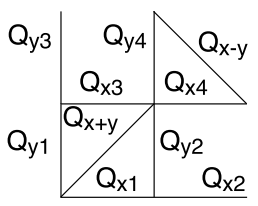

FIG. 1 (color online). (a) Proposed global phase diagram of heavy-fermion metals. Here, $\mathrm{AF}_{s}$ and $\mathrm{AF}_{L}$ refer to antiferromagnetic states without or with static Kondo screening. $P_{L}$ is the paramagnetic heavy Fermi liquid, and $P_{S}$ refers to a paramagnetic phase without static Kondo screening. Adapted with permission from Ref. [7b], Fig. 2, copyright (c) 2010, Wiley-VCH Verlag $\mathrm{GmbH} \& \mathrm{Co} . \mathrm{KGaA}$, Weinheim. We have sketched the proposed trajectory of $\mathrm{Yb}_{2} \mathrm{Pt}_{2} \mathrm{~Pb}$ under magnetic field tuning. (b) ShastrySutherland lattice, denoting the Heisenberg exchange couplings $J_{1}$ on all the horizontal and vertical bonds, and $J_{2}$ along the diagonals. The unit cell is the dashed square, containing four sites $A, B, C, D$. (c) The bond singlet parameters. 
In this work, we study the effect of frustration on the Kondo-Heisenberg model by considering it on the Shastry-Sutherland lattice (SSL) [17], as illustrated in Fig. 1(b). The Hamiltonian is defined as

$$
H=\sum_{(i, j), \sigma} t_{i j}\left(c_{i \sigma}^{\dagger} c_{j \sigma}+\text { H.c. }\right)+J_{K} \sum_{i} \mathbf{S}_{i} \cdot \mathbf{s}_{i}^{c}+\sum_{(i, j)} J_{i j} \mathbf{S}_{i} \cdot \mathbf{S}_{j},
$$

where $(i, j)$ denote the nearest neighbors (NNs) and next nearest neighbors (NNNs) on the SSL as shown in Fig. 1(b). The NN and NNN tight binding parameters for the conduction electrons, denoted by $c_{i \sigma}$, are $t_{1}$ and $t_{2}$, respectively. The spins of the conduction electrons are $\mathbf{s}_{i}^{c}=c_{i \alpha}^{\dagger}\left(\boldsymbol{\sigma}_{\alpha \beta} / 2\right) c_{i \beta}$ at site $i$, where $\sigma_{\alpha \beta}$ are the Pauli spin matrices. They are coupled to spin-1/2 local moments, $\mathbf{S}_{i}$, through an antiferromagnetic Kondo coupling $J_{K}$. We have explicitly included the RKKY interactions, incorporating $J_{1}$ and $J_{2}$, the NN and NNN terms, respectively [Fig. 1(b)]. The degree of frustration is measured by the ratio $G=J_{2} / J_{1}$. We represent the local moments using fermionic spinons $[18,19], f_{i \sigma}$ such that $\mathbf{S}_{i}=f_{i \alpha}^{\dagger}\left(\boldsymbol{\sigma}_{\alpha \beta} / 2\right) f_{i \beta}$ with a constraint $\sum_{\sigma} f_{i \sigma}^{\dagger} f_{i \sigma}=1$ at each lattice site. The spin- $1 / 2$ Heisenberg model on the SSL was extensively studied (e.g., Refs. [17,20-22]). For $J_{2} / J_{1}>2$, it possesses an exact VBS ground state, where singlets form across each disconnected diagonal bond [17]. Whereas for small $J_{2} / J_{1}$, the model has an AF ground state [20,21]. The transition between these two states has not been completely determined [20,21]. The model in the presence of Kondo coupling was studied in some detail by Bernhard et al. [23], and was also discussed qualitatively [8]. As we will discuss, our work here reports the first complete analysis of the relevant phases, and this is essential both in realizing the global phase diagram and in shedding light on the experimentally observed partial Kondo screening (PKS) phase.

Large $-N$ limit.-Generalizing the spin symmetry from $S U(2)$ to $S U(N)$, we arrive at $H=\sum_{(i, j), \sigma} t_{i j}\left(c_{i \sigma}^{\dagger} c_{j \sigma}+\right.$ h.c.) $-J_{K} / N \sum_{i}: B_{i}^{\dagger} B_{i}:-\sum_{(i, j)}\left(J_{i j} / N\right): D_{i j}^{\dagger} D_{i j}$ :, where $B_{i}=\sum_{\sigma} c_{i \sigma}^{\dagger} f_{i \sigma}, D_{i j}=\sum_{\sigma} f_{i \sigma}^{\dagger} f_{j \sigma}$. The sum now runs over $\sigma=1, \ldots, N$, and the constraint becomes $\sum_{\sigma} f_{i \sigma}^{\dagger} f_{i \sigma}=$ $N / 2$. We have also used $: \cdots$ : to denote normal ordering. The large- $N$ mean field Hamiltonian can be expressed as

$$
\begin{aligned}
H_{\mathrm{MF}}= & E-\sum_{(i, j), \sigma}\left(Q_{i j}^{*} f_{i \sigma}^{\dagger} f_{j \sigma}+\text { H.c. }\right)+\sum_{i, \sigma} \lambda_{i} f_{i \sigma}^{\dagger} f_{i \sigma} \\
& +\sum_{(i, j), \sigma} t_{i j}\left(c_{i \sigma}^{\dagger} c_{j \sigma}+\text { H.c. }\right)-\sum_{i, \sigma}\left(b_{i}^{*} c_{i \sigma}^{\dagger} f_{i \sigma}+\text { H.c. }\right) .
\end{aligned}
$$

We have used a Hubbard-Stratonovich transformation decoupling $B_{i}$ and $D_{i j}$ in the Kondo singlet and resonating valence bond (RVB) channels, respectively $[11,24]$, and the constraint is enforced by $\lambda_{i}$. The constant term is $E / N=\sum_{i}\left(\left|b_{i}\right|^{2} / J_{K}-\lambda_{i} / 2\right)+\sum_{(i, j)}\left|Q_{i j}\right|^{2} / J_{i j}$. The Kondo parameter $N b_{i}=J_{K}\left\langle B_{i}\right\rangle$ can be taken to be real by absorbing its phase into the constraint field $\lambda_{i}$ [25], whereas the RVB parameters $N Q_{i j}=J_{i j}\left\langle D_{i j}\right\rangle$ are in general complex.

We solve Eq. (2) by using a four-site unit cell, where each site is labeled by $i \rightarrow(\mathbf{r}, X)$, with $X=A, B, C, D$ marking the sublattice [see Fig. 1(b)], and $\mathbf{r}$ specifying a unit cell. We introduce Fourier transforms per sublattice [26] as $c_{\mathbf{r} X \sigma}=1 / \sqrt{N_{u}} \sum_{\mathbf{k}} e^{-i \mathbf{k} \cdot\left(\mathbf{r}+\boldsymbol{\delta}_{X}\right)} c_{\mathbf{k} X \sigma}$, where $\boldsymbol{\delta}_{X}$ points to each sublattice $X$ from sublattice $A$. Keeping the full generality of the four-site unit cell we introduce sublattice dependent Kondo parameters and constraint fields $b_{X}, \lambda_{X}$, and use ten complex RVB parameters $Q_{i j}$ as shown in Fig. 1(c). These parameters are determined by solving the saddle-point equations self-consistently (see Supplemental Material [27]). We consider the metallic case $0<n_{c}<1$, where $n_{c}=\left(1 / 4 N_{u}\right) \sum_{i, \sigma}\left\langle c_{i \sigma}^{\dagger} c_{i \sigma}\right\rangle$ is the filling of the conduction band.

The zero temperature phase diagram is shown in Fig. 2. Without loss of generality, we have chosen $t_{1} / t_{2}=1.0$ and $n_{c}=0.5$ for Figs. 2 and 3. For small Kondo coupling and a large $J_{2} / J_{1}$ ratio, a VBS ground state arises for which only $Q_{x+y}=Q_{x-y}$ are nonzero. The singlet bonds are the same as in the pure Heisenberg model in the Shastry-Sutherland lattice at large $J_{2} / J_{1}$, and we label it as SSL-VBS. This solution does not break any symmetry of the SSL.

Keeping $J_{K} / t_{1}$ small and decreasing $J_{2} / J_{1}$, we find a first order transition at $J_{2} / J_{1}=1$ from the SSL-VBS to a plaquette VBS (P-VBS) ground state where only $Q_{x 2}=$ $Q_{x 4}=Q_{y 1}=Q_{y 2}$ are nonzero. The P-VBS ground state breaks a reflection symmetry about either of the diagonal bonds in the SSL. It is degenerate with the conventional VBS on the square lattice with only $Q_{x 1}=Q_{x 4}$ being nonzero.

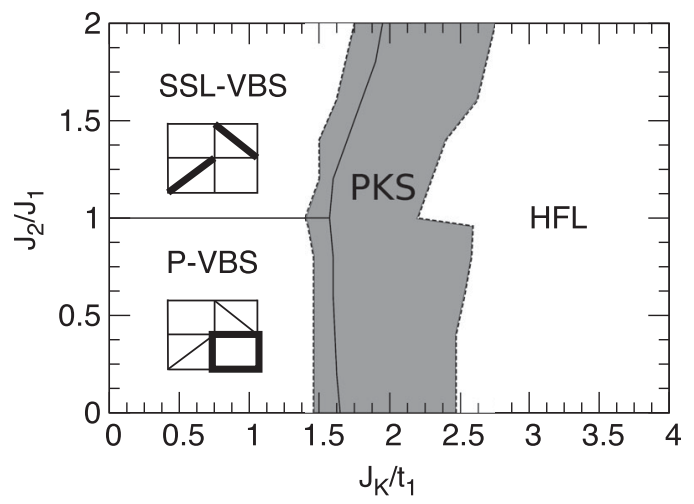

FIG. 2. Large- $N$ phase diagram as a function of frustration $\left(J_{2} / J_{1}\right)$ and Kondo coupling $\left(J_{K} / t_{1}\right)$, for a metallic filling $n_{c}=0.5$. The phases are described in the main text. The solid lines represent first-order transitions, and the dashed lines surrounding the grey area locate the boundaries of the intermediate phases that exhibit partial Kondo screening (PKS). 


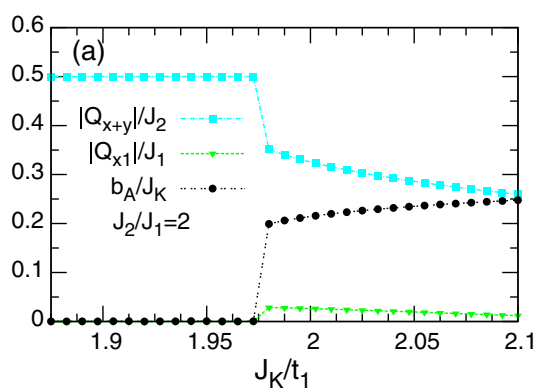

(b)

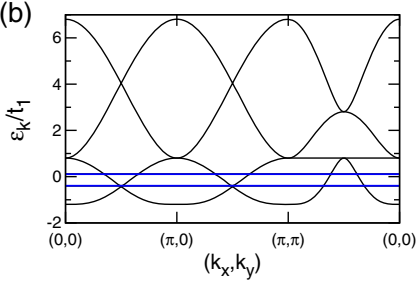

(c)

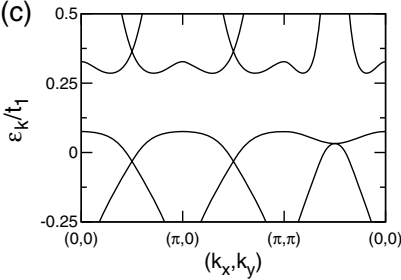

FIG. 3 (color online). (a) The bond and Kondo singlet parameters at a fixed ratio $J_{2} / J_{1}=2.0$ as a function of $J_{K}$, displaying a first order transition from the SSL-VBS to the HFL phase. We show the three dimensionless independent quantities for the solution that breaks no lattice translational symmetry. Band structure along high symmetry directions in the reduced Brillouin zone in the SSL-VBS phase with $J_{K}=0$ and $J_{2} / J_{1}=2.0$ (b) and in the HFL phase with $J_{K}=2.1 t_{1}$ and $J_{2} / J_{1}=2.0$ (c). The thick blue lines are the gapped spinon dispersion in the SSL-VBS phase.

For a large Kondo coupling we find a heavy Fermi liquid (HFL) ground state, which has a nonzero Kondo parameter $b_{A}=b_{B}=b_{C}=b_{D}$. The singlet bond parameters are also nonzero: $Q_{x i}=Q_{y i}$, for $i=1-4$ and $Q_{x+y}=Q_{x-y}$. We also obtain $\lambda_{A}=\lambda_{B}=\lambda_{C}=\lambda_{D}$, so the solution does not break any symmetry of the SSL. Here, we find that each $Q_{i j}$ acquires a finite phase $Q_{i j}=\left|Q_{i j}\right| e^{i \phi_{i j}}$. Correspondingly, we define a gauge independent flux through the triangular and square plaquettes as $\Phi_{\Delta}=\sum_{\Delta} \phi_{i j}(\bmod 2 \pi)$ and $\Phi_{\square}=\sum_{\square} \phi_{i j}(\bmod 2 \pi)$, respectively, where the summation is over the bonds around a plaquette. For the range of fillings $0<n_{c} \lesssim 0.75$, we find $\Phi_{\Delta}=\pi$ and $\Phi_{\square}=0$, whereas for $0.75 \lesssim n_{c}<1$ we obtain $\Phi_{\Delta}=0$ and $\Phi_{\square}=0$. The finite flux through each triangular plaquette is a consequence of the spinons acquiring a finite kinetic energy from their hybridization with the conductionelectron band; we can therefore consider this as a hybridization induced flux phase. However, even though the flux through each triangular plaquette is $\pi$, the total flux through each square plaquette is still zero $(\bmod 2 \pi)$; the flux does not affect the electronic band structure in the HFL phase.

We now turn to the transition among the two VBS phases and the HFL phase. Restricting the solution to these three states, we obtain the phase boundary in Fig. 2 and the mean field parameters shown in Fig. 3(a). Unexpectedly, when considering the general solution we find a number of intermediate states that break the lattice symmetry, in the region shown as the grey shaded area in Fig. 2. In some cases, for example the intermediate phase between the
SSL-VBS phase and the HFL phase, we find a PKS state: some (half) of the moments in the unit cell are still locked into valence bonds, while the other spins are Kondo screened. This is discussed in detail in the Supplemental Material [27]. Tuning $t_{1} / t_{2}$ only affects the location of the phase boundary; a smaller ratio of $t_{1} / t_{2}$ makes the transition between each VBS phase and the HFL phase occur for smaller values of $J_{K} / t_{1}$.

Magnetism at $N=2$.-We now incorporate long range $\mathrm{AF}$ order into our approach. To do so we decouple the Heisenberg term into two distinct channels, but we no longer have access to the large- $N$ limit and are restricted to $N=2$. In keeping with the generalized procedure of Hubbard-Stratonovich decouplings [36], and similar to Ref. [11], we rewrite the Heisenberg term in Eq. (1) as follows: $J_{i j} \mathbf{S}_{i} \cdot \mathbf{S}_{j}=x J_{i j} \mathbf{S}_{i} \cdot \mathbf{S}_{j}+(1-x) J_{i j} \mathbf{S}_{i} \cdot \mathbf{S}_{j} ; \quad$ the term proportional to $x$ is treated within the RVB decoupling described previously. The additional term is decoupled in terms of Néel order: $(1-x) J_{i j} \mathbf{S}_{i} \cdot \mathbf{S}_{j}=(1-x) J_{i j}\left(2 \mathbf{M}_{i}\right.$. $\left.\mathbf{S}_{j}-\mathbf{M}_{i} \cdot \mathbf{M}_{j}\right)$, where $\mathbf{M}_{i}=\left\langle\mathbf{S}_{i}\right\rangle$. We consider the Néel ground state with an ordering wave vector $\mathbf{Q}=(\pi / a, \pi / a)$. This $\mathrm{AF}$ order corresponds to $\mathbf{M}_{A}=\mathbf{M}_{D}=-\mathbf{M}_{C}=$ $-\mathbf{M}_{B}=\mathbf{M}$ within the four site unit cell. In the absence of a Kondo coupling, $J_{K}=0$, the phase diagram of the Heisenberg model as a function of $x$ and $J_{2} / J_{1}$ is presented in the Supplemental Material [27].

The phase diagram at $J_{K}=0$ provides the physical basis for choosing the parameter $x$. Within our fermionic representation, classical AF order arises at $x=0$, corresponding to a full ordered magnetic moment $|\mathbf{M}|=1 / 2$. The ground state wave function of the true quantum $\mathrm{AF}$ state is known to contain considerable RVB correlations [37], suggesting a choice of $x>1 / 2$. Indeed, for the nearest neighbor Heisenberg model $\left(J_{K}=0\right.$ and $\left.J_{2}=0\right)$ we find a quantum AF phase as a self-consistent solution for $x$ in the range $0.67 \leq x<0.8125$, which has a lower free energy than the classical Néel state $\left(Q_{x i}=Q_{y i}=0\right)$. This state, is a free energy local minimum, and taken as a candidate of the true Néel ground state. The quantum AF phase has finite RVB parameters $Q_{x i}=Q_{y i}$ for $i=1-4$, which reduce the ordered moment $|\mathbf{M}|<1 / 2$. Incorporating fluctuations further will reduce the free energy even more, making the AF phase the true ground state in the limit $J_{2} / J_{1} \ll 1$. Here we present the results for $x=0.7$. The phases and the overall profile of the phase diagram are not sensitive to the choice of $x$ in the range $0.67 \leq x<0.8125$, as discussed in the Supplemental Material [27].

The resulting phase diagram is given in Fig. 4, for parameters $n_{c}=0.5$ and $t_{1} / t_{2}=1$. We have restricted the solutions to states that do not break any lattice symmetries. For small $J_{K} / t_{1}$ and tuning the ratio of $J_{2} / J_{1}$, we find a first order transition from the AF phase to the SSL-VBS phase. For small $J_{2} / J_{1}$, and tuning the Kondo coupling, the AF phase has a continuous transition [38] into a spin density wave (SDW) phase characterized by the onset of Kondo 


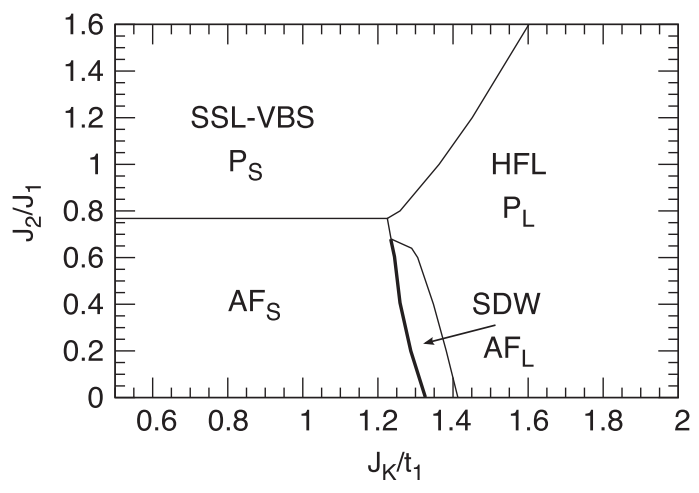

FIG. 4. Phase diagram of the Shastry-Sutherland Kondo lattice incorporating magnetic order for a metallic filling $n_{c}=0.5$. Thin (thick) lines represent first order (continuous [38]) transitions.

screening: $b_{A}=b_{B}=b_{C}=b_{D}$ increases continuously from zero with nonzero values of $\mathbf{M}, Q_{x i}=Q_{y i}$ for $i=$ $1-4$ and $Q_{x+y}=Q_{x-y}$. Upon increasing $J_{K}$ further, there is a first order transition from the SDW phase into the HFL phase with $\mathbf{M}=0$.

Our results demonstrate a rich interplay between Kondo and RKKY interactions. In addition to the AF order and its suppression, there is also the competition between the Kondo effect and VBS order in the magnetically disordered region. In the notation of Fig. 1(a), we associate Kondo hybridization $(b \neq 0)$ with a large Fermi surface (subscript $L)$ and Kondo destruction $(b=0)$ with a small Fermi surface (subscript $S$ ). The phase diagram we have calculated, Fig. 4, represents a remarkable realization of the global phase diagram that had been advanced on qualitative considerations $[7,8]$. It will be instructive to study Kondo lattice models in other geometrically frustrated cases, for example kagome lattices (pertinent to $\mathrm{CePd}_{1-x} \mathrm{Ni}_{x} \mathrm{Al}$ [4]) and triangular lattices (relevant to $\mathrm{YbAgGe}$ [5] and $\mathrm{YbAl}_{3} \mathrm{C}_{3}$ [6]), and explore the generality of the global phase diagram. Compared to those cases, the Kondo model on the SSL has the main advantage that the magnetically frustrated regime is accessible by a large- $N$ approach.

Several remarks are in order. First, in the phase diagram of Fig. 4, we find a line of direct transitions from $\mathrm{AF}_{S}$ to $P_{L}$. However, whether this is a line of transitions or a single point is sensitive to the model parameters in our approach and for $x=0.75$ we find the transition collapsing to a single point (see the Supplemental Material [27]). It is important to consider how further quantum fluctuations will affect the topology of the phase diagram in Fig. 4. Recently, insights have been gained from calculations on a quantum impurity model incorporating local quantum fluctuations [39]; within an extended dynamical mean field context [9], the results of Ref. [39] imply this direct transition to be a line in the phase diagram.

Second, due to an even number of spins per unit cell, the spinon bands are either empty or completely full [8]. Hence, the volume of the Fermi surface will not change when the system goes from the SSL-VBS to the HFL phase. Nonetheless, the topology of the Fermi surfaces reflects the incorporation $(L)$ or absence $(S)$ of the Kondo resonances in the Fermi volume and can be different for the two cases. We show the Fermi surfaces for $n_{c}=0.5$ in the Supplemental Material [27].

Third, it is instructive to compare our results to those of Ref. [23]. Where there is overlap, the results of that work and ours are largely consistent. We are able to draw substantially new implications by studying the competitions of all the phases pertinent to the global phase diagram, including the $\mathrm{AF}_{L}$ phase. Furthermore, our work has also uncovered PKS phases. In a similar vein, we note that the Shastry-Sutherland Kondo lattice was also considered in Ref. [8], with a particular focus on possible superconducting pairings. The implications of our study for superconductivity is an intriguing issue, but is beyond the scope of the present work.

The systematic nature of our results is important not only for generating insights into the global phase diagram, but also to drawing implications for experiments in heavyfermion metals. Our phase diagram in Fig. 4 opens up a trajectory from the $\mathrm{AF}_{S}$ to the HFL phase via a sequence of quantum phase transitions that passes through a VBS, $P_{S}$ phase. This result has implications for $\mathrm{Yb}_{2} \mathrm{Pt}_{2} \mathrm{~Pb}$, where experiments [3] appear to have realized such a sequence of transitions [see Fig. 1(a)].

In addition, we have provided evidence for intermediate, partially Kondo screened phases in metallic cases. This type of phase has also been discussed in a variational quantum Monte Carlo approach in Kondo insulator settings [40]. In this regard, it is intriguing that experiments on the metallic $\mathrm{CePd}_{1-x} \mathrm{Ni}_{x} \mathrm{Al}$ [4] have suggested that the frustration in this material is not large enough to yield a spin liquid, but instead leads to a ground state where some of the magnetic moments form long range AF order, while the others are completely screened by the Kondo effect.

In conclusion, we have studied the global phase diagram in the prototypical geometrically frustrated ShastrySutherland Kondo lattice. Our work represents the first concrete calculation in which all four phases, $\mathrm{AF}_{S}, \mathrm{AF}_{L}$, $P_{S}$, and $P_{L}$ appear in a single zero-temperature phase diagram. Our results have elucidated the rich variety of quantum phases and their transitions in heavy-fermion metals, and provide new insights into the puzzling experimental observations recently made in geometrically frustrated heavy-fermion metals.

We would like to acknowledge useful discussions with D. T. Adroja, M. Aronson, C. H. Chung, P. Coleman, S. Kirchner, A. Nevidomskyy, and E. Nica. This work was supported in part by the NSF Grant No. DMR-1309531 (R. Y. and Q. S.), the U.S. Army Research Office under Grant No. W911NF-14-1-0525, the Robert A. Welch Foundation Grant No. C-1411, the East-DeMarco Fellowship (J. H.P.), and the Alexander von Humboldt 
Foundation (Q.S.). The majority of the calculations have been performed on the Shared University Grid at Rice funded by NSF under Grant EIA-0216467, and a partnership between Rice University, Sun Microsystems, and Sigma Solutions, Inc. Q. S. also acknowledges the hospitality of the the Karlsruhe Institute of Technology, the Aspen Center for Physics (NSF Grant No. 1066293), and the Institute of Physics of Chinese Academy of Sciences. R. Y. was partially supported by the National Science Foundation of China, Grant No. 11374361; the Fundamental Research Funds for the Central Universities; and the Research Funds of Remnin University of China.

*Present address: Condensed Matter Theory Center, Department of Physics, University of Maryland, College Park, Maryland 20742-4111, USA.

[1] L. Balents, Nature (London) 464, 199 (2010).

[2] Q. Si and F. Steglich, Science 329, 1161 (2010).

[3] M. S. Kim and M. C. Aronson, Phys. Rev. Lett. 110, 017201 (2013); M. S. Kim, M. C. Bennett, and M. C. Aronson, Phys. Rev. B 77, 144425 (2008); M. S. Kim and M. C. Aronson, J. Phys. Condens. Matter 23, 164204 (2011).

[4] V. Fritsch, N. Bagrets, G. Goll, W. Kittler, M. J. Wolf, K. Grube, C.-L. Huang, and H. v. Löhneysen, Phys. Rev. B 89, 054416 (2014).

[5] J. K. Dong, Y. Tokiwa, S. L. Bud'ko, P. C. Canfield, and P. Gegenwart, Phys. Rev. Lett. 110, 176402 (2013); S. L. Bud'ko, E. Morosan, and P. C. Canfield, Phys. Rev. B 71, 054408 (2005).

[6] D. D. Khalyavin, D. T. Adroja, P. Manuel, A. DaoudAladine, M. Kosaka, K. Kondo, K. A. McEwen, J. H. Pixley, and Q. Si, Phys. Rev. B 87, 220406(R) (2013).

[7] Q. Si, Physica (Amsterdam) 378B, 23 (2006); Phys. Status Solidi B 247, 476 (2010).

[8] P. Coleman and A. Nevidomskyy, J. Low Temp. Phys. 161, 182 (2010).

[9] Q. Si, S. Rabello, K. Ingersent, and J. L. Smith, Nature (London) 413, 804 (2001).

[10] P. Coleman, C. Pépin, Q. Si, and R. Ramazashvili, J. Phys. Condens. Matter 13, R723 (2001).

[11] T. Senthil, M. Vojta, and S. Sachdev, Phys. Rev. B 69, 035111 (2004).

[12] A. Schröder, G. Aeppli, R. Coldea, M. Adams, O. Stockert, H. v. Löhneysen, E. Bucher, R. Ramazashvili, and P. Coleman, Nature (London) 407, 351 (2000).

[13] S. Friedemann, N. Oeschler, S. Wirth, C. Krellner, C. Geibel, F. Steglich, S. Paschen, S. Kirchner, and Q. Si, Proc. Natl. Acad. Sci. U.S.A. 107, 14547 (2010).

[14] H. Shishido, R. Settai, H. Harima, and Y. Ōnuki, J. Phys. Soc. Jpn. 74, 1103 (2005).

[15] T. Park, F. Ronning, H. Q. Yuan, M. B. Salamon, R. Movshovich, J. L. Sarrao, and J. D. Thompson, Nature (London) 440, 65 (2006).
[16] J. Custers, K.-A. Lorenzer, M. Müller, A. Prokofiev, A. Sidorenko, H. Winkler, A. M. Strydom, Y. Shimura, T. Sakakibara, R. Yu, Q. Si, and S. Paschen, Nat. Mater. 11, 189 (2012).

[17] B. S. Shastry and B. Sutherland, Physica (Amsterdam)) 108B, 1069 (1981.

[18] D. P. Arovas and A. Auerbach Phys. Rev. B 38, 316 (1988).

[19] A. Auerbach, Interacting Electrons and Quantum Magnetism (Springer, New York, 1994).

[20] C. H. Chung, J. B. Marston, and S. Sachdev, Phys. Rev. B 64, 134407 (2001).

[21] A. Lauchli, S. Wessel, and M. Sigrist, Phys. Rev. B 66, 014401 (2002).

[22] S. Miyahara and K. Ueda, J. Phys. Condens. Matter 15, R327 (2003).

[23] B. H. Bernhard, B. Coqblin, and C. Lacroix, Phys. Rev. B 83, 214427 (2011).

[24] P. Coleman and N. Andrei, J. Phys. Condens. Matter 1, 4057 (1989).

[25] N. Read, D. M. Newns, and S. Doniach, Phys. Rev. B 30, 3841 (1984).

[26] S. Furukawa, T. Dodds, and Y. B. Kim, Phys. Rev. B 84, 054432 (2011).

[27] See Supplemental Material at http://link.aps.org/ supplemental/10.1103/PhysRevLett.113.176402, which includes Refs. [28-35], for details on the numerical method, intermediate phases, effect of model parameters, Fermi surfaces, and magnetic phase diagram.

[28] C. G. Broyden, Math. Comput. 19, 577 (1965).

[29] W. H. Press, S. A. Teukolsky, W. T. Vetterling, and B. P. Flannery, Numerical Recipes: The Art of Scientific Computing, 3rd ed. (Cambridge University Press, New York, 2007).

[30] D. D. Johnson, Phys. Rev. B 38, 12807 (1988).

[31] L. D. Marks and D. R. Luke, Phys. Rev. B 78, 075114 (2008).

[32] A. Baran, A. Bulgac, M. Forbes, G. Hagen, W. Nazarewicz, N. Schunck, and M. Stoitsov, Phys. Rev. C 78, 014318 (2008).

[33] R. Žitko, Phys. Rev. B 80, 125125 (2009).

[34] S. Yunoki, E. Dagotto, S. Costamagna, and J. A. Riera, Phys. Rev. B 78, 024405 (2008).

[35] R. Yu, P. Goswami, Q. Si, P. Nikolic, and J.-X. Zhu, Nat. Commun. 4, 2783 (2013).

[36] J. W. Negele and H. Orland, Quantum Many-Particle Systems (Westview Press, Boulder, CO, 1998).

[37] E. Dagotto and A. Moreo, Phys. Rev. B 38, 5087(R) (1988).

[38] We are not able to discern between a continuous and a very weakly first order transition.

[39] E. M. Nica, K. Ingersent, J. X. Zhu, and Qimiao Si, Phys. Rev. B 88, 014414 (2013).

[40] Y. Motome, K. Nakamikawa, Y. Yamaji, and M. Udagawa, Phys. Rev. Lett. 105, 036403 (2010). 